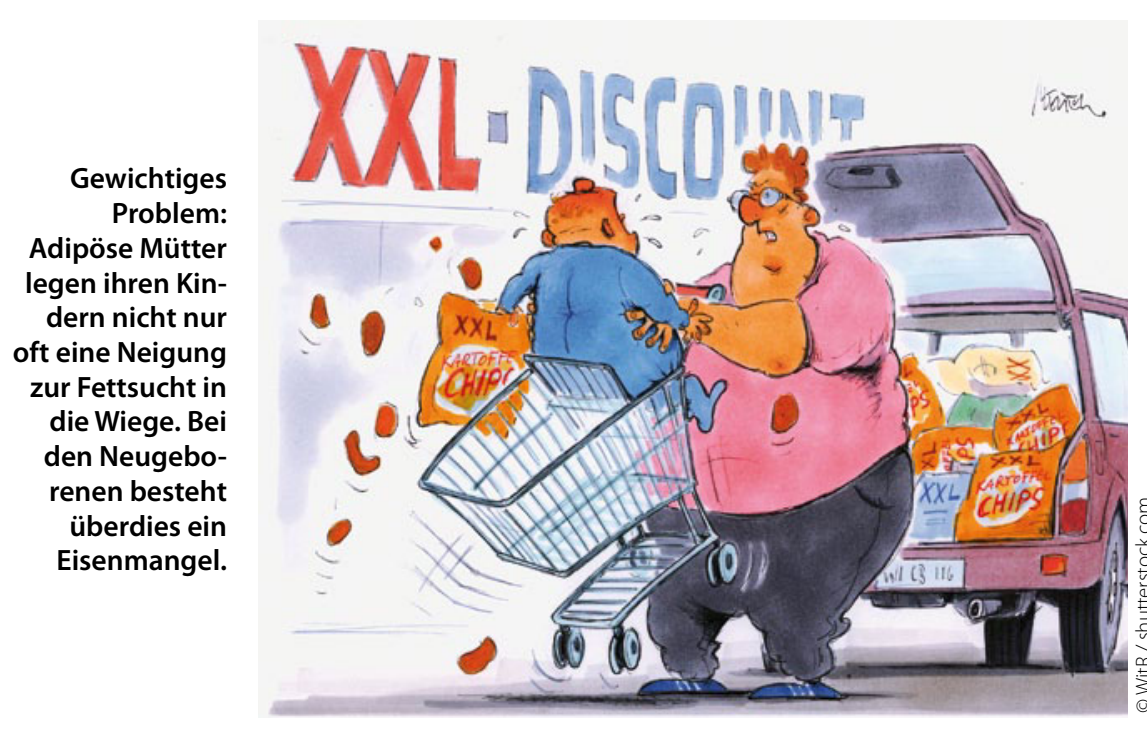

HIRNENTWICKLUNG BEEINTRÄCHTIGT?

\section{Übergewicht der Mutter kostet Kinder Eisen}

— Kinder, deren Mütter stark übergewichtig sind, erhalten in der Spätschwangerschaft zu wenig Eisen. Dies könnte die Hirnentwicklung beeinträchtigen, wie Forscher auf dem Jahrestreffen der Pediatric Academic Societies in Denver berichtet haben.

Die Arbeitsgruppe um Prof. Pamela Kling hatte 281 Paare von Müttern mit ihren Kindern untersucht. Ein BMI $\geq 30$ der Mutter wurde als Adipositas definiert. Den Eisenspiegel der Neugeborenen bestimmten die Forscher anhand von Parametern im Nabelschnurblut.
Wie die Messungen zeigten, war starkes Übergewicht der Mutter mit Beeinträchtigungen des neonatalen Eisenstatus verbunden. „Unsere Resultate sprechen dafür, dass der Eisentransfer in der Spätschwangerschaft reduziert ist", resümieren Kling und ihr Team. In dieser Phase vervierfacht sich der Eisenbedarf. Kinder müssen die Hälfte allen Eisens, das sie fürs Wachsen brauchen, noch vor ihrer Geburt einsammeln.

$\mathrm{RB}$.

Kling PJ et al. Maternal Obesity and Delivery: A Risk Factor for Newborn Iron Deficiency. PAS Annual Meeting 2011, Abstract 1380.8
KHK-PATIENTEN

\section{Der Bauch bestimmt das Risiko}

— Nicht der BMI, sondern der Umfang von Bauch und Hüfte sind entscheidend für das Mortalitätsrisiko von Herzpatienten. Dies stellte die Sichtung der Daten von insgesamt 15923 Patienten aus fünf weltweiten Studien klar. Die Auswertungen der amerikanischen Mayo-Klinik ergaben für KHKPatienten mit stammbetonter Fettsucht ein besonders hohes Mortalitätsrisiko (HR $1,7)$. Selbst bei Menschen mit normalem BMI waren die mit Bäuchlein stärker gesundheitsgefährdet (HR: 1,7) als Schlanke. Der BMI dagegen verhielt sich umgekehrt proportional zu einer höheren Sterbewahrscheinlichkeit (HR: 0,64).

So scheint der BMI keine ideale Maßeinheit für das Mortalitätsrisiko zu sein. Vor allem sagt er nichts über die Verteilung des Körperfetts aus. Offenbar beeinflusst das metabolisch aktivere Viszeralfett das Moralitätsrisikos stärker als der Speck, der sich an anderen Körperstellen sammelt. Damit ergibt sich für den Hausarzt eine sehr einfache und schnelle Methode, das Risiko zu erfassen. Gleichzeitig hat er ein Argument mehr, seinen KHK-Patienten eine gesundheitsfördernde Maßnahme in Form einer Diät ans Herz zu legen, deren Erfolg er jederzeit sofort nachmessen kann.

CS .

Coutinho, Tet al. Central Obesity and Survival in Subjects With Coronary Artery Disease. J Am Coll Cardiol 2011, 57: 1877-1886, doi: 10.1016/j.jacc.2010.11.058

\section{Buch-Tipp: Von Aua bis Zipperlein}

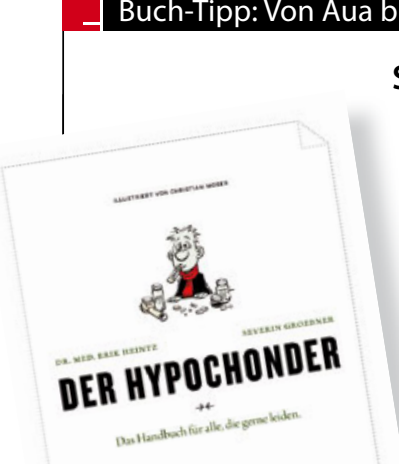

\section{Sind wir nicht alle ein bisschen Hypochonder?}

Das Titelbild dieser MMW-Ausgabe entstammt dem Buch „Der Hypochonder - Das Handbuch für alle, die gerne leiden“ von Dr. Erik Heintz. Unterstützt wurde er bei der Bearbeitung dieses Themas vom Wiener Kabarettisten Severin Groebner und dem Comic-Zeichner und Illustrator Christian Moser. „Erfreulicherweise ist den Autoren die schmale Gratwanderung zwischen geziemender Ernsthaftigkeit und einer erfrischend humorvollen Betrachtungsweise gelungen. Das Buch kann daher auch Betroffenen empfohlen werden, solange die Tugend der Selbstironie noch nicht ganz erloschen ist", so MMW-Rezensent Dr. Peter Stiefelhagen. Mit Tucholsky empfiehlt er: „Umarmen Sie dieses Buch, und lesen Sie den „Hypochonder“! Es wird Sie begeistern, denn es verspricht bei diesem traurigen Thema eine wohl dosierte Stimmungsaufhellung, um nicht zu sagen eine psychohygienische Wirkung."

Die vollständige Rezension, Interviews mit den Autoren etc. finden Sie unter www.springermedizin.de/hypochonder-handbuch.

Weitere Illustrationen von Christian Moser finden Sie unter www.monster-des-alltags.de 